\title{
Estimating the Modified Weibull Parameters in Presence of Constant-Stress Partially Accelerated Life Testing
}

\author{
Mohamed A. W. Mahmoud \\ Mathematics Department, Faculty of Science, Al-Azhar University, Nasr City (11884), Cairo, Egypt. \\ mawmahmoud11@yahoo.com \\ Rashad M. EL-Sagheer \\ Mathematics Department, Faculty of Science, Al-Azhar University, Nasr City (11884), Cairo, Egypt. \\ rashadmath@yahoo.com,rashadmath@azhar.edu.eg \\ Amr M. Abou-Senna \\ Mathematics Department, Faculty of Engineering, Shoubra, Benha University, Cairo, Egypt. \\ amr.abosenna@yahoo.com
}

Received 14 July 2017

Accepted 10 January 2018

\begin{abstract}
Accelerated life testing is very important in life testing experiments because it saves time and cost. In this paper, assuming that the lifetime of items under use condition follows the modified Weilbull distribution, partially accelerated life tests based on progressive Type-II censored samples are considered. The likelihood equations are to be solved numerically to obtain the maximum likelihood estimates. Based on normal approximation to the asymptotic distribution of maximum likelihood estimates, the approximate confidence intervals for the parameters are derived. Two bootstrap confidence intervals called bootstrap-p and bootstrap-t are also discussed. It is difficult to get explicit form for Bayes estimates, so we use Markov chain Monte Carlo method to solve this problem, which gives us flexibility to construct the credible intervals for parameters. Finally, a simulation study is performed to compare between MLEs and Bayes estimates.
\end{abstract}

Keywords: Modified Weilbull distribution; Constant-partially accelerated life testing; Progressive Type-II censoring; Asymptotic confidence intervals; Bootstrap; Bayesian estimation.

2000 Mathematics Subject Classification: 62No5; 62F10.

$\begin{array}{llll}\text { ALT } & \text { Accelerated Life Test } & \text { PRO-II-C } & \text { Progressive Type-II Censored } \\ \text { PALT } & \text { Partially ALT } & \text { MCMC } & \text { Markov chain Monte Carlo } \\ \text { C-SPALT } & \text { Constant-stress PALT } & \text { MWD } & \text { Modified Weilbull Distribution } \\ \text { SF } & \text { Reliability Function } & \text { PDF } & \text { Probability Density Function } \\ \text { HRF } & \text { Hazard Rate Function } & \text { CDF } & \text { Cumulative Distribution Function } \\ \text { BP } & \text { Percentile Bootstrap } & \text { MLEs } & \text { Maximum Likelihood Estimates } \\ \text { BT } & \text { Bootstrap-t } & \text { ACIs } & \text { Approximate Confidence Intervals } \\ \text { CRIs } & \text { credible intervals } & \text { M-H } & \text { Metropolis-Hastings method }\end{array}$




\section{Introduction}

There is a difficulity in getting information about the lifetime of products with high quality during testing under normal conditions, so ALTs are used in manufacturing industries to get failure data in short period of time. According to Nelson [24] there exist three ALT methods. The first one is called constant-stress ALT, where the stress is being constant during the experiment $[3,5,4,18]$. The second one is called progressive-stress ALT, where the applied stress is increasing during time [6,31] . The third one is called step-stress ALT,where the test condition changes at a certain time or after the termination of specific number of failures. Miler and Nelson [23] got the optimal step-stress ALT plans in the case where tested items have exponentially distributed lives and are observed until all tests fail. For more researches on ALTs, see others [14, 17, 20, 25, 28, 32]. In ALTs, the test items are tested at accelerated conditions, by applying higher levels of stress, to induce failures. Data collected at such accelerated conditions are then extrapolated through statistical model to estimate the lifetime distribution at normal condition. We use PALT when the acceleration factor is unknown, where items are examined at both normal and accelerated conditions. The stress in PALT can be divided into 3-types which are constant-stress, step-stress and progressive-stress. In step-stress PALT, items are tested at normal level, then the stress will be changed at a certain time. Many authers have dealt with this type, including Abdel-Hamid and Al-Hussaini [2].In C-SPALT (which is the main topic of this paper ) we run each item at either normal or accelerated condition only. In life testing experiments, our target is to save time and cost and this leads us to what is known as censoring in which complete data about failure times for all experimental units may not be obtained, but data obtained from these experiments are called censored data. The most common censoring schemes are Type-I censoring and Type-II censoring, but in these two types the withdrawing of units is not allowed until the end of the experiment, so a more general censoring scheme called PRO-II-C has been used in this paper to overcome this problem in which the removal of prespecified number of units is done when an individual unit fails, this continues until fixed number of units fail, at which stage the remainder of the surviving units are removed. Another generalization of Weibull distribution called MWD was introduced by Xie et al. [33] and a detailed statistical analysis was given in Tang et al. [29]. The distribution is in fact a generalization of the model studied by Chen [9]. If $X$ follows a MWD, then the PDF, CDF, SF and HRF are given respectively by

$$
\begin{gathered}
f_{1}(x)=\lambda \alpha\left(\frac{x}{\beta}\right)^{\alpha-1} \exp \left\{\left(\frac{x}{\beta}\right)^{\alpha}+\lambda \beta\left(1-\exp \left[\left(\frac{x}{\beta}\right)^{\alpha}\right]\right)\right\}, \quad x>0, \alpha, \beta, \lambda>0, \\
F_{1}(x)=1-\exp \left\{\lambda \beta\left(1-\exp \left\{\left(\frac{x}{\beta}\right)^{\alpha}\right\}\right)\right\}, \quad x>0, \alpha, \beta, \lambda>0, \\
S_{1}(x)=\exp \left\{\lambda \beta\left(1-\exp \left\{\left(\frac{x}{\beta}\right)^{\alpha}\right\}\right)\right\},
\end{gathered}
$$

and

$$
h_{1}(x)=\lambda \alpha\left(\frac{x}{\beta}\right)^{\alpha-1} \exp \left\{\left(\frac{x}{\beta}\right)^{\alpha}\right\} .
$$

The shape of $h_{1}(x)$ of MWD depends only on the shape parameter $\alpha$, see [19].

- For $\alpha \geq 1: h_{1}(x)$ is an increasing function . 
- For $0<\alpha<1: h_{1}(x)$ is decreasing for $x<\beta\left(\frac{1}{\alpha}-1\right) \frac{1}{\alpha}$ and increasing for $x>\beta\left(\frac{1}{\alpha}-1\right) \frac{1}{\alpha}$.

The hazard rate plots of the distribution are given in Fig.1.

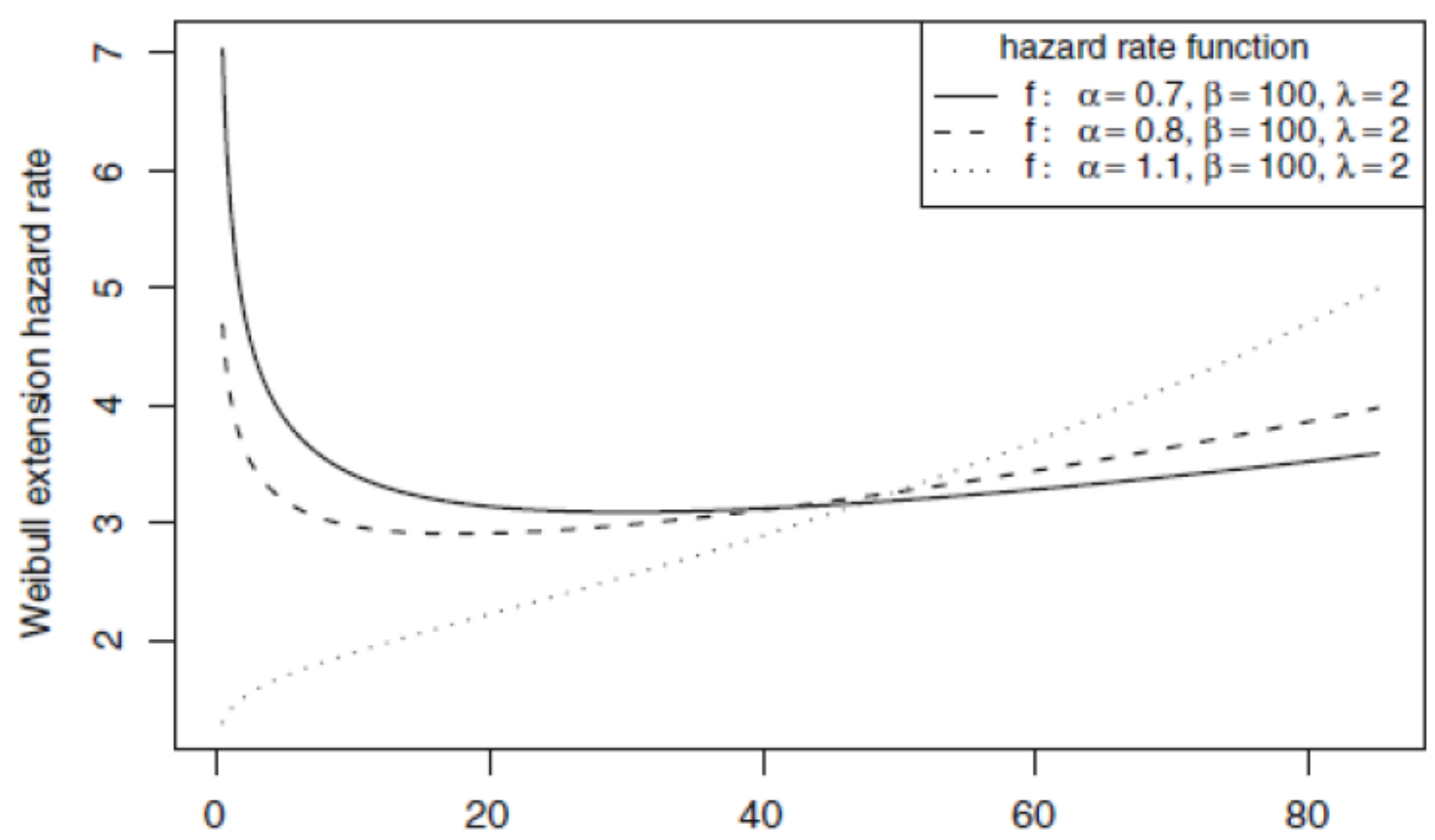

Fig. 1. Weibull extension hazard rate functions

It is clear that the exponential power distribution $(\alpha, \beta)$ is a special case of MWD with $\lambda=1$, so any results obtained in our study, will also be valid for the exponential Power distribution $(\alpha, \beta)$ and this was considered and studied by Smith and Bain [27]. The MWD model has been fitted to the failure times of Aarset data (Aarset [1]) and the failure times of a sample of devices in a large system Gupta et al. [15].The detailed description of the data set can be found in Meeker and Escobar [21]

This paper can be organized as follows: In Section 2 some basic assumptions and description of the model are presented. The derivation of the MLEs of the parameters as well as the corresponding ACIs and the two parametric bootstrap confidence intervals for the parameters are showed in Section 3. Section 4 discusses the Bayesian approach that uses the well-known MCMC method.A numerical example to depict the approach is given in Section 5. A simulation study is presented in Section 6. Finally, some concluding remarks are considered in Section 7.

\section{Model description and assumptions}

In C-SPALT, $n_{1}$ items are randomly selected among $n$ test items and tested under use condition and $n_{2}=n-n_{1}$ remaining items are subjected to an accelerated condition. PRO-II-C is applied as follows, when the first failure occurs, $X_{k 1 ; m_{k}, n_{k}}^{R_{k}}, R_{k 1}$ items are randomly withdrawn from the remaining $n_{k}-1$ surviving items. At the second failure $X_{k 2 ; m_{j}, n_{j}}^{R_{k}}, R_{k 2}$ items from the remaining $n_{k}-2-R_{k 1}$ items are randomly withdrawn. The test continues until the $m_{k} t h$ failure $X_{k m_{k} ; m_{k}, n_{k}}^{R_{k}}$ at which time, all remaining $R_{k m_{k}}=n_{k}-m_{k}-\sum_{t=1}^{m_{k}-1} R_{k t}$ items are withdrawn for $k=1,2$. If the failure times of the $n_{k}$ items originally in the test are from a continuous population with $\operatorname{CDF} F_{k}(x)$ and $\operatorname{PDF} f_{k}(x)$, the 
joint PDF for $X_{k 1 ; m_{k}, n_{k}}^{R_{k}}<X_{k 2 ; m_{k}, n_{k}}^{R_{k}}<\ldots<X_{k m_{k} ; m_{k}, n_{k}}^{R_{k}}$ and $k=1,2$ ( see Balakrishnan and Aggarwala $[7]$ ) is given by

$$
L(\alpha, \beta, \lambda, \mu \mid \underline{x})=\prod_{k=1}^{2} B_{k}\left\{\prod_{z=1}^{m_{k}} f_{k}\left(x_{k z ; m_{k}, n_{k}}\right)\left(S_{k}\left(x_{k z ; m_{k}, n_{k}}\right)\right)^{R_{k z}}\right\},
$$

where

$$
B_{k}=n_{k}\left(n_{k}-1-R_{k 1}\right)\left(n_{k}-2-R_{k 1}-R_{k 2}\right) \cdots\left(n_{k}-m_{k}-\sum_{t=1}^{m_{k}-1} R_{k t}\right) .
$$

Now, we assume that the lifetime of the items tested at use condition follows a MWD with PDF,CDF,SF and HRF given in (1.1)-(1.4). The hazard rate of an item tested at accelerated condition is given by $h_{2}(x)=\mu h_{1}(x)$, where $\mu$ is an acceleration factor satisfying $\mu>1$. Therefore, the HRF, SF, CDF and PDF under acceerated condition are given respectively by

$$
\begin{gathered}
h_{2}(x)=\mu \lambda \alpha\left(\frac{x}{\beta}\right)^{\alpha-1} \exp \left\{\left(\frac{x}{\beta}\right)^{\alpha}\right\} \\
S_{2}(x)=\exp \left[-\int_{0}^{x} h_{2}(z) d z\right]=\exp \left\{\lambda \mu \beta\left(1-\exp \left\{\left(\frac{x}{\beta}\right)^{\alpha}\right\}\right)\right\}, \\
F_{2}(x)=1-\exp \left\{\lambda \mu \beta\left(1-\exp \left\{\left(\frac{x}{\beta}\right)^{\alpha}\right\}\right)\right\},
\end{gathered}
$$

and

$$
f_{2}(x)=\lambda \mu \alpha\left(\frac{x}{\beta}\right)^{\alpha-1} \exp \left\{\left(\frac{x}{\beta}\right)^{\alpha}+\lambda \beta \mu\left(1-\exp \left\{\left(\frac{x}{\beta}\right)^{\alpha}\right\}\right)\right\}
$$

\section{Maximum likelihood estimation}

Let $X_{k 1 ; m_{k}, n_{k}}^{R_{k}}<X_{k 2 ; m_{k}, n_{k}}^{R_{k}}<\cdots<X_{k m_{k} ; m_{k}, n_{k}}^{R_{k}}$ for $k=1,2$ denote two PRO-II-C samples from two populations whose CDFs and PDFs are given in (1.1), (1.2), (2.4) and (2.5) with $R_{k}=$ $\left(R_{k 1}, R_{k 2}, \ldots, R_{k m_{k}}\right)$. The likelihood function without normalized constant can be written as

$$
\begin{aligned}
L(\alpha, \beta, \lambda, \mu \mid \underline{x})= & (\alpha \lambda)^{m_{1}+m_{2}} \mu^{m_{2}} \prod_{k=1}^{2} \prod_{z=1}^{m_{k}}\left(\frac{x_{k z}}{\beta}\right)^{\alpha-1} \\
& \times \exp \left\{\sum_{k=1}^{2} \sum_{z=1}^{m_{k}}\left(\frac{x_{k z}}{\beta}\right)^{\alpha}+\lambda \beta \xi_{k}\left(1+R_{k z}\right) \Psi x_{k z}\right\},
\end{aligned}
$$

where

$$
\Psi x_{k z}=1-\exp \left\{\left(\frac{x_{k z}}{\beta}\right)^{\alpha}\right\}
$$

and

$$
\xi_{k}= \begin{cases}1, & \text { if } k=1 \\ \mu, & \text { if } k=2\end{cases}
$$


Therefore, the $\log$-likelihood function $\ell(\alpha, \beta, \lambda, \mu \mid \underline{x})=\log L(\alpha, \beta, \lambda, \mu \mid \underline{x})$ without normalized constant is given by

$$
\begin{aligned}
\ell(\alpha, \beta, \lambda, \mu \mid \underline{x})= & \sum_{k=1}^{2} \sum_{z=1}^{m_{k}}\left(\left(\frac{x_{k z}}{\beta}\right)^{\alpha}+(\alpha-1) \log x_{k z}+\lambda \beta \xi_{k}\left(1+R_{k z}\right) \Psi x_{k z}\right) \\
& +m_{2} \log \mu+\left(m_{1}+m_{2}\right) \log \left(\frac{\lambda \alpha}{\beta^{\alpha-1}}\right) .
\end{aligned}
$$

Then, we differentiate (3.3) with respect to $\alpha, \beta, \lambda$ and $\mu$ and equate each result to zero. For simplification, we can write $\frac{\partial \ell(\alpha, \beta, \lambda, \mu \mid \underline{x})}{\partial \alpha}, \frac{\partial \ell(\alpha, \beta, \lambda, \mu \mid \underline{x})}{\partial \beta}, \frac{\partial \ell(\alpha, \beta, \lambda, \mu \mid \underline{x})}{\partial \lambda}$ and $\frac{\partial \ell(\alpha, \beta, \lambda, \mu \mid \underline{x})}{\partial \mu}$ as $\frac{\partial \ell}{\partial \alpha}, \frac{\partial \ell}{\partial \beta}, \frac{\partial \ell}{\partial \lambda}$ and $\frac{\partial \ell}{\partial \mu}$ respectively

$$
\begin{gathered}
\frac{\partial \ell}{\partial \alpha}=\sum_{k=1}^{2} \sum_{z=1}^{m_{k}}\left(\frac{x_{k z}}{\beta}\right)^{\alpha} \log \left(\frac{x_{k z}}{\beta}\right)\left(1-\lambda \beta \xi_{k}\left(1+R_{k z}\right) \exp \left\{\left(\frac{x_{k z}}{\beta}\right)^{\alpha}\right\}\right)+\log x_{k z} \\
+\left(m_{1}+m_{2}\right)\left(\frac{1}{\alpha}-\log \beta\right)=0 \\
\frac{\partial \ell}{\partial \beta}=\sum_{k=1}^{2} \sum_{z=1}^{m_{k}} \lambda \xi_{k}\left(1+R_{k z}\right)\left(1-\exp \left\{\left(\frac{x_{k z}}{\beta}\right)^{\alpha}\right\}\right) \\
+\sum_{k=1}^{2} \sum_{z=1}^{m_{k}} \alpha\left(\frac{x_{k z}}{\beta}\right)^{\alpha}\left(\lambda \xi_{k}\left(1+R_{k z}\right) \exp \left\{\left(\frac{x_{k z}}{\beta}\right)^{\alpha}\right\}-\frac{1}{\beta}\right) \\
-\frac{\left(m_{1}+m_{2}\right)(\alpha-1)}{\beta}=0 \\
\frac{\partial \ell}{\partial \lambda}=\frac{\left(m_{1}+m_{2}\right)}{\lambda}+\sum_{k=1}^{2} \sum_{z=1}^{m_{k}} \xi_{k} \beta \Psi x_{k z}\left(1+R_{k z}\right)=0
\end{gathered}
$$

and

$$
\frac{\partial \ell}{\partial \mu}=\frac{m_{2}}{\mu}+\sum_{z=1}^{m_{2}} \lambda \beta\left(1-\exp \left\{\left(\frac{x_{2 z}}{\beta}\right)^{\alpha}\right\}\right)\left(1+R_{2 z}\right)=0
$$

A system of nonlinear simultaneous equations in four unknowns vaiables $\alpha, \beta, \lambda$ and $\mu$ is resulted, but it is not an easy task to get an exact solution, so we tackle this situation by using a numerical method such as Newton-Raphson to obtain an approximate solution of the above nonlinear system, see EL-Sagheer [13].

\subsection{Approximate interval estimation}

Upon forming the Fisher information matrix, we take the inverse of it to get the asymptotic variances and covariances of the MLE for parameters $\alpha, \beta, \lambda$ and $\mu$, but unfortunately, the exact mathematical expressions are very difficult to obtain, so we give an approximate asymptotic varaince-covariance 
matrix for the MLE by ignoring the expectation operator E.

$$
\begin{aligned}
& F^{-1}=\left[\begin{array}{cccc}
-\frac{\partial^{2} \ell}{\partial \alpha^{2}} & -\frac{\partial^{2} \ell}{\partial \alpha \partial \beta} & -\frac{\partial^{2} \ell}{\partial \alpha \partial \lambda} & -\frac{\partial^{2} \ell}{\partial \alpha \partial \mu} \\
\frac{-\partial^{2} \ell}{\partial \beta \partial \alpha} & -\frac{\partial^{2} \ell}{\partial \beta^{2}} & -\frac{\partial^{2} \ell}{\partial \beta \partial \lambda} & -\frac{\partial^{2} \ell}{\partial \beta \partial \mu} \\
-\frac{\partial^{2} \ell}{\partial \lambda \partial \alpha} & -\frac{\partial^{2} \ell}{\partial \lambda \partial \beta} & -\frac{\partial^{2} \ell}{\partial \lambda^{2}} & -\frac{\partial^{2} \ell}{\partial \lambda \partial \mu} \\
-\frac{\partial^{2} \ell}{\partial \mu \partial \alpha} & -\frac{\partial^{2} \ell}{\partial \mu \partial \beta} & -\frac{\partial^{2} \ell}{\partial \mu \partial \lambda} & -\frac{\partial^{2} \ell}{\partial \mu^{2}}
\end{array}\right]_{\downarrow(\widehat{\alpha}, \widehat{\beta}, \widehat{\lambda}, \widehat{\mu})}^{-1}
\end{aligned}
$$

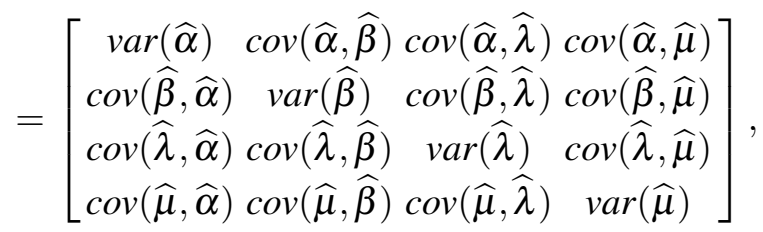

where

$$
\begin{gathered}
\frac{\partial^{2} \ell}{\partial \alpha^{2}}=\sum_{k=1}^{2} \sum_{z=1}^{m_{k}}\left(\frac{x_{k z}}{\beta}\right)^{\alpha}\left(\log \left(\frac{x_{k z}}{\beta}\right)\right)^{2}\left(1-\lambda \beta \xi_{k}\left(1+R_{k z}\right)\right) \exp \left\{\left(\frac{x_{k z}}{\beta}\right)^{\alpha}\right\} \Phi x_{k z} \\
-\frac{\left(m_{1}+m_{2}\right)}{\alpha^{2}}, \\
\Phi x_{k z}=1+\left(\frac{x_{k z}}{\beta}\right)^{\alpha}, \\
\frac{\partial^{2} \ell}{\partial \beta^{2}}=\sum_{k=1}^{2} \sum_{z=1}^{m_{k}}\left(\frac{\lambda \alpha}{\beta} \xi_{k}\left(1+R_{k z}\right)\left(\frac{x_{k z}}{\beta}\right)^{\alpha} \exp \left\{\left(\frac{x_{k z}}{\beta}\right)^{\alpha}\right\}\left(1-\alpha-\alpha\left(\frac{x_{k z}}{\beta}\right)^{\alpha}\right)\right) \\
+\sum_{k=1}^{2} \sum_{z=1}^{m_{k}}\left(\frac{\alpha}{\beta^{2}}(1+\alpha)\left(\frac{x_{k z}}{\beta}\right)^{\alpha}\right)+\frac{\left(m_{1}+m_{2}\right)(\alpha-1)}{\beta^{2}} \\
\frac{\partial^{2} \ell}{\partial \lambda^{2}}=-\frac{\left(m_{1}+m_{2}\right)}{\lambda^{2}},
\end{gathered}
$$

and

$$
\frac{\partial^{2} \ell}{\partial \mu^{2}}=-\frac{m_{2}}{\mu^{2}}
$$

The common method to construct the confidence interval for the parameters $\alpha, \beta, \lambda$ and $\mu$ is to use the asymptotic normal distribution of MLEs and this is showed by Vander Weil and Meeker [30]. Therefore, $(1-\gamma) 100 \%$ confidence intervals for parameters $\alpha, \beta, \lambda$ and $\mu$ become

$$
\widehat{\alpha} \pm Z_{\gamma / 2} \sqrt{\operatorname{var}(\widehat{\alpha})}, \quad \widehat{\beta} \pm Z_{\gamma / 2} \sqrt{\operatorname{var}(\widehat{\beta})}, \quad \widehat{\lambda} \pm Z_{\gamma / 2} \sqrt{\operatorname{var}(\widehat{\lambda})}, \quad \widehat{\mu} \pm Z_{\gamma / 2} \sqrt{\operatorname{var}(\widehat{\mu})}
$$

where $Z_{\gamma / 2}$ is the percentile of the standard normal distribution with right-tail probability $\gamma / 2$. 


\subsection{Bootstrap confidence intervals}

The bootstrap is a resampling method for statistical inference and commonly used to estimate CIs. For more information about parametric and non-parametric bootstrap methods, see $[10,12]$. We propose to use confidence intervals based on the parameteric bootstrap methods (i) BP method based on the idea of Efron [11], (ii) BT method based on the idea of Hall [16]. The algorithms for estimating the confidenc eintervals using both methods are illustrated below.

\subsubsection{BP method}

Step1: From the original data $\underline{x} \equiv\left(X_{k 1 ; m_{k}, n_{k}}^{R_{k}}<X_{k 2 ; m_{k}, n_{k}}^{R_{k}}<\cdots<X_{k m_{k} ; m_{k}, n_{k}}^{R_{k}}\right)$ for $k=1,2$ compute the MLEs of the parameters: $\widehat{\alpha}, \widehat{\beta}, \widehat{\lambda}$ and $\widehat{\mu}$ by solving the nonlinear Equations (3.4)-(3.7).

Step2: Use $\widehat{\alpha}, \widehat{\beta}, \widehat{\lambda}$ and $\widehat{\mu}$ to generate two independant PRO-II-C samples of size $m_{1}$ and $m_{2}$ from the MWD using algorithm presented in Balakrishnan and Sandhu [8].

Step3: As in Step 1, based on $\underline{x}^{*}$ compute the bootstrap sample estimates of $\widehat{\alpha}, \widehat{\beta}, \widehat{\lambda}$ and $\widehat{\mu}$, say $\widehat{\alpha}^{*}, \widehat{\beta}^{*}, \widehat{\lambda}^{*}$ and $\widehat{\mu}^{*}$.

Step4: Repeat Steps 2-3 $N$ times representing $N$ bootstrap MLE's of $\alpha, \beta, \lambda$ and $\mu$ based on $N$ different bootstrap samples.

Step5: Arrange all $\widehat{\alpha}^{* \prime} s, \widehat{\beta}^{* \prime} s, \widehat{\lambda}^{* \prime} s$ and $\widehat{\mu}^{* \prime} s$, in an ascending order to obtain the bootstrap sample $\left(\hat{\eta}_{j}^{*[1]}, \hat{\eta}_{j}^{*[2]}, \ldots, \hat{\eta}_{j}^{*[N]}\right), j=1,2,3,4$ where $\eta_{1}^{*}=\alpha^{*}, \eta_{2}^{*}=\beta^{*}, \eta_{3}^{*}=\lambda^{*}, \eta_{4}^{*}=\mu^{*}$.

Let $G(z)=P\left(\hat{\eta}_{j}^{*} \leq z\right)$ be the CDF of $\hat{\eta}_{j}^{*}$. Define $\hat{\eta}_{j b o o t}^{*}=G^{-1}(z)$ for given $z$. The approximate bootstrap $100(1-\gamma) \%$ confidence interval of $\hat{\eta}_{j}^{*}$ is given by

$$
\left[\hat{\eta}_{j b o o t}^{*}\left(\frac{\gamma}{2}\right), \quad \hat{\eta}_{j b o o t}^{*}\left(\frac{1-\gamma}{2}\right)\right]
$$

\subsubsection{BT method}

Step 1: From the original data $\underline{x} \equiv\left(X_{k 1 ; m_{k}, n_{k}}^{R_{k}}<X_{k 2 ; m_{k}, n_{k}}^{R_{k}}<\cdots<X_{k m_{k} ; m_{k}, n_{k}}^{R_{k}}\right)$ for $k=1,2$ compute the MLEs of the parameters: $\widehat{\alpha}, \widehat{\beta}, \widehat{\lambda}$ and $\widehat{\mu}$ by solving the nonlinear Equations (3.4)-(3.7).

Step 2: Using $\widehat{\alpha}, \widehat{\beta}, \widehat{\lambda}$ and $\widehat{\mu}$ generate a bootstrap sample $\left\{x_{1}^{*}, x_{2}^{*}, \ldots, x_{n}^{*}\right\}$. Based on $\left\{x_{1}^{*}, x_{2}^{*}, \ldots, x_{n}^{*}\right\}$ compute the bootstrap estimate of $\alpha, \beta, \lambda$ and $\mu$, say $\widehat{\alpha}^{*}, \widehat{\beta}^{*}, \widehat{\lambda}^{*}$ and $\widehat{\mu}^{*}$ and the following statistics

$$
T_{j}^{*}=\frac{\sqrt{n}\left(\widehat{\eta}_{j}^{*}-\widehat{\eta}_{j}\right)}{\sqrt{\operatorname{Var}\left(\widehat{\eta}_{j}^{*}\right)}},
$$

where $\hat{\eta}_{1}=\hat{\alpha}, \hat{\eta}_{2}=\hat{\beta}, \hat{\eta}_{3}=\hat{\lambda}$ and $\hat{\eta}_{4}=\hat{\mu}$ for $j=1,2,3,4$ and $\operatorname{Var}\left(\widehat{\eta}_{j}^{*}\right)$ is obtained using the Fisher information matrix.

Step 3: Repeat Step 2, $\mathrm{N}$ boot times.

For the $T_{1}^{*}, T_{2}^{*}, T_{3}^{*}$ and $T_{4}^{*}$ values obtained in Step2, determine the upper and lower bounds of the $100(1-\gamma) \%$ confidence interval of $\alpha, \beta, \lambda$ and $\mu$ as follows: let $H(x)=P\left(T_{j}^{*} \leq x\right), j=1,2,3,4$ 
be the $\mathrm{CDF}$ of $T_{1}^{*}, T_{2}^{*}, T_{3}^{*}$ and $T_{4}^{*}$. For a given $x$, define

$$
\widehat{\eta}_{j B o o t-t}(x)=\widehat{\eta}_{j}+n^{-1 / 2} \sqrt{\operatorname{Var}\left(\widehat{\eta}_{j}\right)} H^{-1}(x)
$$

The $100(1-\gamma) \%$ ACI of $\hat{\eta}_{j}$ is given by

$$
\left(\widehat{\eta}_{j B o o t-t}\left(\frac{\gamma}{2}\right), \quad \widehat{\eta}_{j B o o t-t}\left(1-\frac{\gamma}{2}\right)\right)
$$

\section{Bayesian estimation using MCMC}

In this section we describe how to obtain the Bayes estimates and the corresponding credible intervals of parameters $\alpha, \beta, \lambda$ and $\mu$ when they are unknown. In some situations where we do not have sufficient prior information, we can use non-informative uniform distribution as the prior distribution. . The joint posterior density will then be in proportion to the likelihood function. Here we consider the prior distributions for our parameters $\alpha, \beta, \lambda$ and $\mu$ are

$$
\begin{array}{ll}
\pi(\alpha) \propto \alpha^{-1} & , \alpha>0, \\
\pi(\beta) \propto \beta^{-1} & , \beta>0, \\
\pi(\lambda) \propto \lambda^{-1} & , \lambda>0 \\
\pi(\mu) \propto \mu^{-1} & , \mu>1 .
\end{array}
$$

Therefore, the joint prior of the four parameters can be expressed by

$$
\pi(\alpha, \beta, \lambda, \mu) \propto(\alpha, \beta, \lambda, \mu)^{-1}, \alpha>0, \beta>0, \lambda>0, \mu>1 .
$$

The joint posterior density function of $\alpha, \beta, \lambda$ and $\mu$ given the data ,denoted by $\pi^{*}(\alpha, \beta, \lambda \mu \mid \underline{x})$ can be written as

$$
\pi^{*}(\alpha, \beta, \lambda, \mu \mid \underline{x})=\frac{\ell(\alpha, \beta, \lambda, \mu \mid \underline{x}) \times \pi(\alpha, \beta, \lambda, \mu)}{\int_{0}^{\infty} \int_{0}^{\infty} \int_{0}^{\infty} \int_{0}^{\infty} \ell(\alpha, \beta, \lambda, \mu \mid \underline{x}) \times \pi(\alpha, \beta, \lambda, \mu) d \alpha d \beta d \lambda d \mu} .
$$

It is not possible to compute (4.2) analytically. Therefore, we propose the MCMC technique to generate samples from the posterior distributions and then compute the Bayes estimate for each individual parameter. There are many MCMC schemes and it is difficult to choose among them, but an important sub-class of MCMC methods are Gibbs sampling and more general Metropoliswithin-Gibbs sampler.

\subsection{The Metropolis-Hastings -Within-Gibbs sampling}

We propose to use Gibbs sampling procedure to generate a sample from the posterior density function $\pi^{*}(\alpha, \beta, \lambda, \mu \mid x)$ and in turn compute the Bayes estimates and also construct the corresponding credible intervals based on the generated posterior sample. In order to use the method of MCMC for estimating the parameters, namely, $\alpha, \beta, \lambda$ and $\mu$, let us consider independent priors (4.1) for the parameters $\alpha, \beta, \lambda$ and $\mu$. The expression for the posterior is directly proportional to the likelihood 
and the prior and this can be written as

$$
\begin{aligned}
\pi^{*}(\alpha, \beta, \lambda, \mu \mid \underline{x}) \propto & \mu^{m_{2}-1} \lambda^{m_{1}+m_{2}-1} \alpha^{m_{1}+m_{2}-1} \beta^{-1} \prod_{k=1}^{2} \prod_{z=1}^{m_{k}}\left(\frac{x_{k z}}{\beta}\right)^{\alpha-1} \\
& \times \exp \left\{\sum_{k=1}^{2} \sum_{z=1}^{m_{k}}\left(\frac{x_{k z}}{\beta}\right)^{\alpha}+\lambda \beta \xi_{k}\left(1+R_{k z}\right) \Psi x_{k z}\right\} .
\end{aligned}
$$

The conditional posterior densities of $\lambda, \mu, \alpha$ and $\beta$ are as follows:

$$
\begin{gathered}
\pi_{1}^{*}(\lambda \mid \alpha, \beta, \mu, \underline{x}) \sim \operatorname{Gamma}\left(m_{1}+m_{2}, \sum_{k=1}^{2} \sum_{z=1}^{m_{k}} \beta \xi_{k}\left(1+R_{k z}\right) \Psi\left(x_{k z}\right)\right), \\
\pi_{2}^{*}(\mu \mid \alpha, \beta, \lambda, \underline{x}) \sim \operatorname{Gamma}\left(m_{2}, \sum_{z=1}^{m_{2}} \beta \lambda\left(1+R_{2 z}\right) \Psi x_{2 z}\right), \\
\pi_{3}^{*}(\alpha \mid \beta, \lambda, \mu, \underline{x}) \propto \alpha^{m_{1}+m_{2}-1} \prod_{k=1}^{2} \prod_{z=1}^{m_{k}}\left(\frac{x_{k z}}{\beta}\right)^{\alpha-1} \\
\quad \times \exp \left\{\sum_{k=1}^{2} \sum_{z=1}^{m_{k}}\left(\frac{x_{k z}}{\beta}\right)^{\alpha}-\lambda \beta \xi_{k}\left(1+R_{k z}\right) \exp \left\{\left(\frac{x_{k z}}{\beta}\right)^{\alpha}\right\}\right\},
\end{gathered}
$$

and

$$
\begin{aligned}
\pi_{4}^{*}(\beta \mid \alpha, \lambda, \mu, \underline{x}) \propto & \beta^{-1} \prod_{k=1}^{2} \prod_{z=1}^{m_{k}}\left(\frac{x_{k z}}{\beta}\right)^{\alpha-1} \\
& \times \exp \left\{\sum_{k=1}^{2} \sum_{z=1}^{m_{k}}\left(\frac{x_{k z}}{\beta}\right)^{\alpha}+\lambda \beta \xi_{k}\left(1+R_{k z}\right) \Psi x_{k z}\right\} .
\end{aligned}
$$

We note that Equations (4.4) and (4.5) follow gamma distribution, so samples of $\lambda$ and $\mu$ can be easily generated using any gamma generating routine.The posterior of $\alpha$ in Equation (4.6) and the posterior of $\beta$ in Equation (4.7) are unknown, but if we plot each of them, their graphs are similar to normal distribution, see Figures 3 and 4, so we use the Metropolis-Hastings (M-H) method with normal proposal distribution to generate from each of them. For more details about M-H algorithm, see [22] and [26].

The algorithm of Gibbs sampling is as follows :

Step 1: Start with an $\left(\beta^{(0)}=\hat{\beta}, \alpha^{(0)}=\hat{\alpha}, \lambda^{(0)}=\hat{\lambda}, \mu^{(0)}=\hat{\mu}\right)$.

Step 2: Set $t=1$.

Step 3: Generate $\lambda^{(t)}$ from $\pi_{1}^{*}\left(\lambda \mid \alpha^{(t-1)}, \beta^{(t-1)}, \mu^{(t-1)}, x\right)$.

Step 4: Generate $\mu^{(t)}$ from $\pi_{2}^{*}\left(\mu \mid \alpha^{(t-1)}, \beta^{(t-1)}, \lambda^{(t)}, x\right)$.

Step 5: Using M-H method, generate $\alpha^{(t)}$ from $\pi_{3}^{*}(\alpha \mid \beta, \lambda, \mu, x)$ with proposal distribution $\mathrm{N}\left(\alpha^{(t-1)}, \sigma^{2}\right)$.

Step 6: Using M-H method, generate $\beta^{(t)}$ from $\pi_{4}^{*}(\beta \mid \alpha, \lambda, \mu, x)$ with proposal distribution $\mathrm{N}\left(\beta^{(t-1)}, \sigma^{2}\right)$.

Step 7: Compute $\alpha^{(t)}, \beta^{(t)}, \lambda^{(t)}$ and $\mu^{(t)}$. 
Step 8: Set $t=t+1$.

Step 9: Repeat Steps 3-6 N times.

Step 10: Obtain the Bayes MCMC point estimate of $\eta_{j}$ where $\eta_{1}=\alpha, \eta_{2}=\beta, \eta_{3}=\lambda$ and $\eta_{4}=\mu$ for $j=1,2,3$ and 4 as

$$
E\left(\eta_{j} \mid \text { data }\right)=\frac{1}{N-M} \sum_{k=L+1}^{N} \eta_{j}^{(k)}
$$

where $M$ is the burn-in period .

Step 11: To compute CRIs of $\eta_{j}$, order $\eta_{j}^{(M+1)}, \eta_{j}^{(M+2)}, \ldots, \eta_{j}^{(N)}$ as $\eta_{j(1)}<\eta_{j(2)}<\ldots<\eta_{j(N-M)}$. Then the $100(1-2 \gamma) \%$ symmetric CRI becomes

$$
\left(\eta_{j(\gamma(N-L))}, \eta_{j((1-\gamma)(N-L))}\right)
$$

\section{Data analysis}

In this section, we show a numerical example to check estimation procedures. We generate two samples from the modified weilbull distribution with parameters $(\alpha, \beta, \lambda, \mu)=(10,0.06,2,2)$ using progressive type-II censoring scheme with $R_{1}=(3,1,1,2,2,1,3,0,1,5,2,2,2,1,1,2,1,0$, $0,0), R_{2}=(2,0,2,0,2,0,2,0,2,0,0,0,2,0,0,3,0,1,0,1,0,1,0,0,2,0,0,0,0,0), m_{1}=20$, $m_{2}=30$ and $n_{1}=n_{2}=50$. The MLEs, bootstrap and bayes point estimates have been calculated for the parameters $\alpha, \beta, \lambda$ and $\mu$ and listed in Table 1 . Also we computed 95\% ACI, PBCIs, BTCIs and CRIs for the parameters and listed them in Tables 2 and 3 . In MCMC procedure, the chain has been run 12000 and the first 2000 have been canceled as burn-in . Figure 1 shows the PDFs under normal and accelerated conditions.

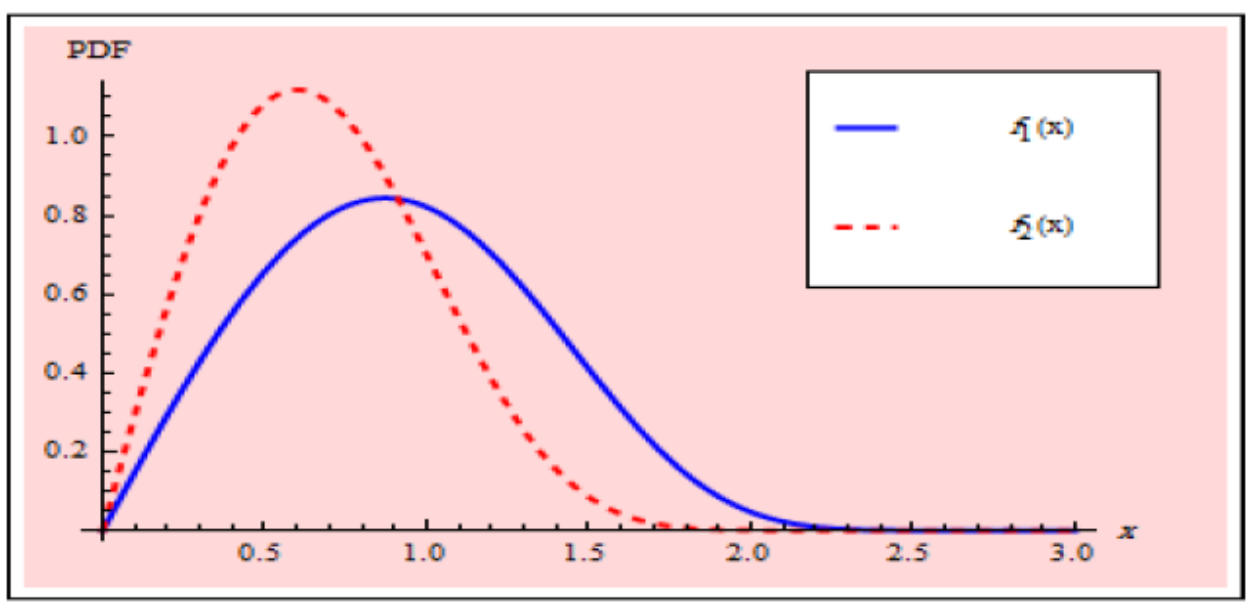

Fig. 2. PDFs under normal and accelerated conditions 


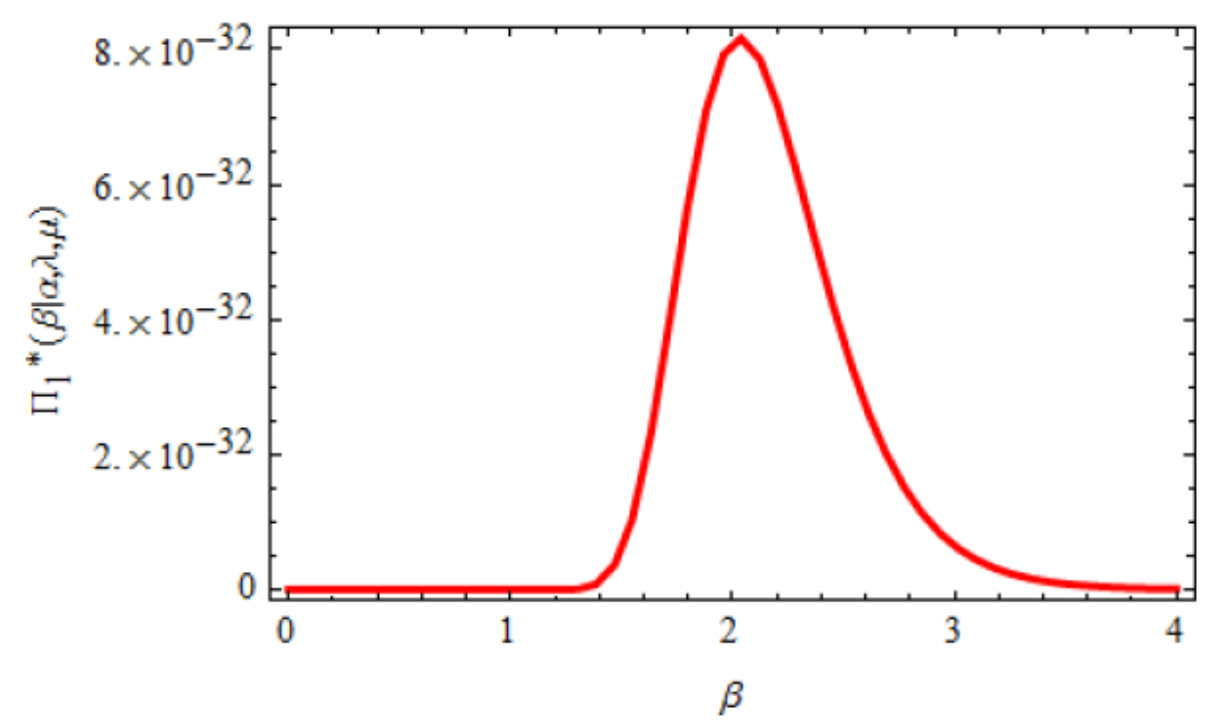

Fig. 3. Posterior density function of $\beta$

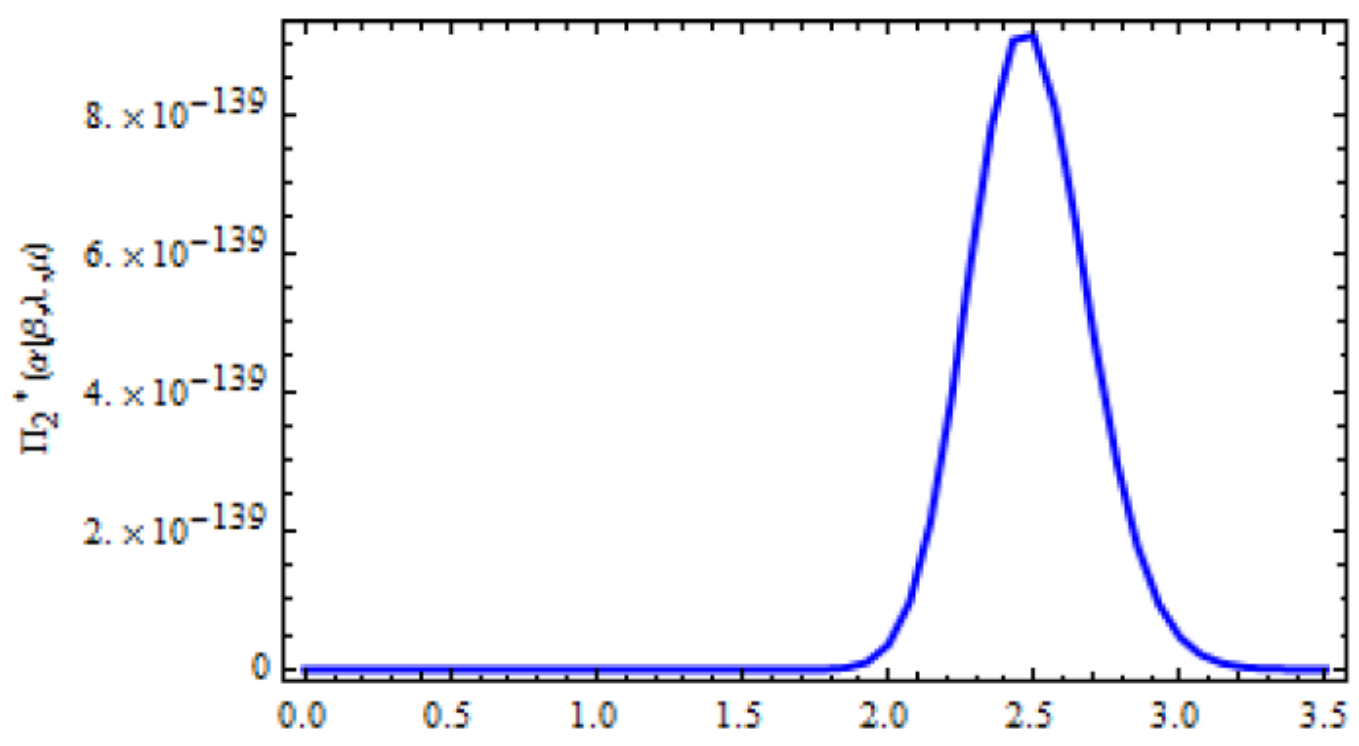

Fig. 4. Posterior density function of $\alpha$

Table 1. Point estimates for parameters $(\alpha, \beta, \lambda, \mu)=(10,0.06,2,2)$.

\begin{tabular}{ccccc}
\hline Parameter & ML & BP & BT & MCMC \\
\hline$\alpha$ & 10.6137 & 10.0452 & 11.1165 & 11.0597 \\
$\beta$ & 0.0591 & 0.0579 & 0.0602 & 0.0604 \\
$\lambda$ & 1.9059 & 1.9693 & 1.8338 & 1.8501 \\
$\mu$ & 2.7211 & 2.7930 & 2.2309 & 2.1600 \\
\hline
\end{tabular}


Table $2.95 \%$ Confidence intervals for parameters $\alpha$ and $\beta$.

\begin{tabular}{lcccc}
\hline Method & $\alpha$ & Length & $\beta$ & Length \\
\hline ACI & {$[-13.125,34.3524]$} & 47.4774 & {$[0.0323,0.0859]$} & 0.0536 \\
BPCI & {$[5.8844,11.8860]$} & 6.0017 & {$[0.0490,0.1009]$} & 0.0519 \\
BTCI & {$[5.6916,14.6896]$} & 8.9980 & {$[0.0351,0.0862]$} & 0.0511 \\
CRI & {$[4.3584,13.0047]$} & 8.6463 & {$[0.0302,0.0774]$} & 0.0472 \\
\hline
\end{tabular}

Table $3.95 \%$ Confidence intervals for parameters $\lambda$ and $\mu$.

\begin{tabular}{lcccc}
\hline Method & $\lambda$ & Length & $\mu$ & Length \\
\hline ACI & {$[-8.5964,11.4082]$} & 20.0047 & {$[1.1039,4.3383]$} & 3.2345 \\
BPCI & {$[0.1319,2.7786]$} & 2.6466 & {$[1.4858,4.4691]$} & 2.9833 \\
BTCI & {$[0.1121,3.5866]$} & 3.4745 & {$[0.0532,2.8848]$} & 2.8315 \\
CRI & {$[1.4251,3.0219]$} & 1.5968 & {$[1.6561,4.4182]$} & 2.7621 \\
\hline
\end{tabular}

\section{Numerical comparison}

In this section, we made a simulation study using MATHEMATICA ver. 8.0 and computed the average point estimates (AVG) and the mean square error (MSE) for the parameters $\alpha, \beta, \lambda$ and $\mu$ . The procedure was performed 1000 times and the population parameters were $(\alpha, \beta, \lambda, \mu)=(10$, $0.06,2,2)$ with sample sizes $n_{1}=n_{2}=n$ and $m_{1}=m_{2}=m$ and results are shown in Table 4.

$$
A V G=\frac{1}{M} \sum_{i=1}^{M} \hat{\xi}_{k}^{(i)}, \quad M S E=\frac{1}{M} \sum_{i=1}^{M}\left(\hat{\xi}_{k}^{(i)}-\xi_{k}\right)^{2}
$$

where $k=1,2,3,4$ and $\xi_{1}=\alpha, \xi_{2}=\beta, \xi_{3}=\lambda, \xi_{4}=\mu$. In our study, three different censoring schemes (CSs) are considered. Scheme I, where all the units are withdrawn when the first failure occurs . In scheme II, all the units are withdrawn in the middle of the experiment when half of the tested units had been censored. The last one is scheme III, where all the units are withdrawn at the end of the experiment when the last failure occurs. For constant $\alpha=1$, the AVG and MSE for the parameters $(\beta, \lambda, \mu)=(0.06,2,2)$ with sample sizes $n_{2}=2 n_{1}=2 n$ are computed and results are shown in Table 5. Also, $95 \%$ CIs are calculated and coverage probability is estimated by counting the number of CIs that covered the true values and divide by 1000, while the average credible interval length is calculated for each parameter by summing the lengths of intervals in each iteration and divide by 1000 and results are shown in Table 6 . The same procedures are repeated again, but for constant $\beta=1$ and results are shown in Tables 7 and 8 . 
Table 4. AVG and MSE of ML and Bayes estimates for $(\alpha, \beta, \lambda, \mu)=(10,0.06,2,2)$.

\begin{tabular}{|c|c|c|c|c|c|c|c|c|c|}
\hline \multirow[b]{2}{*}{$(n, m)$} & \multirow[b]{2}{*}{ CS } & \multicolumn{3}{|c|}{ MLE } & \multirow[b]{2}{*}{$\mu$} & \multirow[b]{2}{*}{$\alpha$} & \multicolumn{2}{|c|}{ MCMC } & \multirow[b]{2}{*}{$\mu$} \\
\hline & & $\alpha$ & $\beta$ & $\lambda$ & & & $\beta$ & $\lambda$ & \\
\hline \multirow[t]{6}{*}{$(30,15)$} & I & 9.261 & 0.059 & 1.953 & 1.699 & 8.908 & 0.058 & 2.117 & 1.846 \\
\hline & & $(0.545)$ & $(0.000005)$ & $(0.002)$ & $(0.09)$ & (1.191) & $(0.000005)$ & $(0.013)$ & $(0.023)$ \\
\hline & II & 10.149 & 0.059 & 1.644 & 2.327 & 8.257 & 0.059 & 2.277 & 1.988 \\
\hline & & $(0.562)$ & $(0.0011)$ & $(0.295)$ & $(0.513)$ & (4.118) & $(0.0097)$ & $(0.82)$ & $(0.083)$ \\
\hline & III & 10.396 & 0.059 & 1.741 & 2.483 & 7.531 & 0.059 & 3.14 & 1.907 \\
\hline & & (0.694) & $(0.008)$ & $(0.418)$ & $(0.687)$ & (9.942) & (0.009) & $(2.47)$ & $(0.126)$ \\
\hline \multirow[t]{6}{*}{$(30,25)$} & I & 10.269 & 0.059 & 1.830 & 2.215 & 9.465 & 0.059 & 2.237 & 2.138 \\
\hline & & $(0.522)$ & $(0.006)$ & $(0.263)$ & $(0.32)$ & $(0.962)$ & $(0.006)$ & $(0.45)$ & $(0.213)$ \\
\hline & II & 10.211 & 0.059 & 1.682 & 2.33 & 9.060 & 0.058 & 2.243 & 2.140 \\
\hline & & $(0.636)$ & (0.008) & (0.309) & $(0.413)$ & (1.481) & $(0.008)$ & $(0.466)$ & $(0.182)$ \\
\hline & III & 10.234 & 0.059 & 1.814 & 2.299 & 8.697 & 0.058 & 2.529 & 2.021 \\
\hline & & (0.636) & $(0.008)$ & $(0.234)$ & $(0.317)$ & (2.784) & (0.008) & $(0.723)$ & (0.064) \\
\hline \multirow[t]{6}{*}{$(50,25)$} & I & 10.336 & 0.059 & 1.751 & 2.293 & 9.356 & 0.059 & 2.233 & 2.153 \\
\hline & & (0.709) & $(0.008)$ & $(0.321)$ & $(0.416)$ & (1.103) & $(0.008)$ & $(0.552)$ & $(0.181)$ \\
\hline & II & 10.144 & 0.059 & 1.618 & 2.272 & 8.576 & 0.059 & 2.201 & 2.001 \\
\hline & & $(0.562)$ & $(0.001)$ & $(0.255)$ & $(0.326)$ & (3.156) & $(0.001)$ & $(0.323)$ & $(0.093)$ \\
\hline & III & 10.362 & 0.059 & 1.791 & 2.372 & 9.156 & 0.058 & 2.377 & 2.143 \\
\hline & & (0.704) & $(0.007)$ & $(0.423)$ & $(0.552)$ & (2.505) & $(0.0076)$ & $(0.970)$ & (0.179) \\
\hline \multirow[t]{6}{*}{$(50,40)$} & I & 10.178 & 0.058 & 1.814 & 2.234 & 9.723 & 0.0589 & 2.036 & 2.222 \\
\hline & & $(0.620)$ & (0.008) & $(0.342)$ & $(0.266)$ & (1.421) & $(0.008)$ & $(0.705)$ & $(0.235)$ \\
\hline & II & 10.056 & 0.059 & 1.877 & 2.183 & 9.760 & 0.05 & 2.025 & 2.189 \\
\hline & & $(0.426)$ & $(0.006)$ & $(0.237)$ & $(0.145)$ & $(0.405)$ & (0.006) & $(0.265)$ & (0.151) \\
\hline & III & 10.239 & 0.059 & 1.857 & 2.250 & 9.882 & 0.058 & 2.028 & 2.237 \\
\hline & & $(0.557)$ & $(0.006)$ & (0.207) & (0.198) & $(0.432)$ & (0.006) & $(0.202)$ & (0.168) \\
\hline \multirow[t]{6}{*}{$(100,50)$} & I & 10.094 & 0.059 & 1.833 & 2.184 & 9.773 & 0.0599 & 1.968 & 2.196 \\
\hline & & $(0.380)$ & $(0.005)$ & $(0.243)$ & (0.209) & $(0.615)$ & $(0.0056)$ & $(0.263)$ & $(0.201)$ \\
\hline & II & 10.038 & 0.059 & 1.822 & 2.176 & 9.841 & 0.0597 & 1.913 & 2.186 \\
\hline & & $(0.407)$ & (0.006) & (0.187) & (0.193) & $(0.380)$ & $(0.006)$ & $(0.160)$ & $(0.195)$ \\
\hline & III & 10.132 & 0.059 & 1.817 & 2.304 & 9.879 & 0.059 & 1.932 & 2.292 \\
\hline & & $(0.412)$ & (0.004) & $(0.127)$ & $(0.267)$ & $(0.349)$ & $(0.059)$ & (0.097) & $(0.230)$ \\
\hline \multirow[t]{6}{*}{$(100,75)$} & I & 10.095 & 0.059 & 1.860 & 2.154 & 10.017 & 0.059 & 1.892 & 2.190 \\
\hline & & $(0.430)$ & $(0.005)$ & $(0.145)$ & (0.098) & $(0.395)$ & $(0.005)$ & $(0.135)$ & (0.124) \\
\hline & II & 10.068 & 0.059 & 1.893 & 2.079 & 9.855 & 0.058 & 1.975 & 2.066 \\
\hline & & $(0.412)$ & $(0.006)$ & $(0.158)$ & $(0.103)$ & $(0.360)$ & $(0.006)$ & $(0.149)$ & $(0.093)$ \\
\hline & III & 10.081 & 0.059 & 1.798 & 2.138 & 9.998 & 0.059 & 1.828 & 2.184 \\
\hline & & $(0.367)$ & $(0.005)$ & $(0.153)$ & $(0.096)$ & $(0.344)$ & $(0.005)$ & $(0.141)$ & $(0.120)$ \\
\hline
\end{tabular}


Table 5. AVG and MSE of ML and Bayes estimates for $(\beta, \lambda, \mu)=(0.06,2,2)$.

\begin{tabular}{|c|c|c|c|c|c|c|c|}
\hline \multicolumn{8}{|c|}{ at constant $\alpha=1$} \\
\hline \multirow[b]{2}{*}{$\left(n, m_{1}, m_{2}\right)$} & \multirow[b]{2}{*}{ CS } & \multicolumn{2}{|c|}{ MLE } & \multirow[b]{2}{*}{$\mu$} & \multicolumn{3}{|c|}{ MCMC } \\
\hline & & $\beta$ & $\lambda$ & & $\beta$ & $\lambda$ & $\mu$ \\
\hline \multirow[t]{6}{*}{$(20,10,20)$} & I & 0.0595 & 2.2412 & 1.768 & 0.0813 & 3.0334 & 1.874 \\
\hline & & $(0.000039)$ & $(0.175)$ & $(0.1137)$ & $(0.000736)$ & (1.496) & $(0.0729)$ \\
\hline & II & 0.0634 & 2.398 & 1.711 & 0.0815 & 2.9633 & 1.8591 \\
\hline & & $(0.000099)$ & $(0.2586)$ & $(0.1414)$ & $(0.02459)$ & (1.4942) & (0.1167) \\
\hline & III & 0.0706 & 2.3604 & 1.6939 & 0.2053 & 3.5787 & 1.7144 \\
\hline & & $(0.00049)$ & $(0.2413)$ & $(0.1518)$ & $(0.12144)$ & (3.2679) & $(0.1341)$ \\
\hline \multirow[t]{6}{*}{$(20,15,30)$} & I & 0.0612 & 2.3479 & 1.7185 & 0.0594 & 2.9502 & 1.7814 \\
\hline & & $(0.000079)$ & $(0.212)$ & $(0.141)$ & $(0.02294)$ & (1.7419) & (0.117) \\
\hline & II & 0.0639 & 2.4036 & 1.7455 & 0.0662 & 2.9805 & 1.7973 \\
\hline & & $(0.000096)$ & $(0.2523)$ & $(0.1202)$ & $(0.02091)$ & (1.4225) & $(0.1003)$ \\
\hline & III & 0.0633 & 2.3429 & 1.6993 & 0.1016 & 3.1577 & 1.8746 \\
\hline & & $(0.000127)$ & $(0.2425)$ & $(0.1485)$ & $(0.0038)$ & (1.8259) & (2.0778) \\
\hline \multirow[t]{6}{*}{$(40,20,40)$} & I & 0.0635 & 2.4268 & 1.7509 & 0.0495 & 2.8796 & 1.796 \\
\hline & & $(0.000071)$ & $(0.2902)$ & $(0.1265)$ & $(0.06141)$ & (1.1537) & (0.1106) \\
\hline & II & .0625 & 2.3693 & 1.718 & 0.071 & 2.6171 & 1.7737 \\
\hline & & $(0.000084)$ & $(0.2228)$ & $(0.1288)$ & $(0.000283)$ & $(0.5426)$ & $(0.1037)$ \\
\hline & III & 0.07 & 2.4429 & 1.7695 & 0.1848 & 3.1837 & 2.1503 \\
\hline & & $(0.00027)$ & $(0.2714)$ & (0.1078) & $(0.16457)$ & (1.835) & (5.229) \\
\hline \multirow[t]{6}{*}{$(40,30,60)$} & I & 0.0621 & 2.3523 & 1.7305 & 0.0677 & 2.6087 & 1.7623 \\
\hline & & $(0.000049)$ & $(0.2032)$ & $(0.1134)$ & $(0.000127)$ & (0.4954) & $(0.0966)$ \\
\hline & II & 0.0631 & 2.4219 & 1.7492 & 0.0689 & 2.6488 & 1.7817 \\
\hline & & $(0.00052)$ & $(0.2576)$ & $(0.0887)$ & $(0.00015)$ & $(0.5372)$ & $(0.075)$ \\
\hline & III & 0.0656 & 2.4295 & 1.7411 & 0.0805 & 2.8295 & 1.7528 \\
\hline & & $(0.000107)$ & $(0.26)$ & $(0.1005)$ & $(0.000823)$ & (0.8777) & (0.094) \\
\hline \multirow[t]{6}{*}{$(60,30,60)$} & I & 0.0631 & 2.4061 & 1.7402 & 0.0689 & 2.6615 & 1.7766 \\
\hline & & $(0.000045)$ & $(0.2409)$ & $(0.1055)$ & $(0.000147)$ & $(0.5708)$ & $(0.0923)$ \\
\hline & II & 0.061 & 2.2998 & 1.7173 & 0.061 & 2.49 & 1.7463 \\
\hline & & $(0.0000447)$ & $(0.1542)$ & $(0.1222)$ & $(0.000117)$ & $(0.3257)$ & $(0.1072)$ \\
\hline & III & 0.0682 & 2.4393 & 1.7343 & 0.0895 & 2.9274 & 1.7261 \\
\hline & & $(0.000181)$ & $(0.2578)$ & $(0.1097)$ & $(0.01906)$ & (1.091) & $(0.1126)$ \\
\hline \multirow[t]{6}{*}{$(60,50,90)$} & I & 0.0627 & 2.4049 & 1.707 & 0.066 & 2.5637 & 1.7263 \\
\hline & & $(0.000037)$ & $(0.2314)$ & $(0.1114)$ & $(0.000081)$ & (0.4084) & (0.1004) \\
\hline & II & 0.0628 & 2.3958 & 1.7377 & 0.0662 & 2.5464 & 1.7538 \\
\hline & & $(0.000031)$ & $(0.2098)$ & $(0.0942)$ & (0.000069) & $(0.3607)$ & $(0.0875)$ \\
\hline & III & 0.0638 & 2.4202 & 1.6941 & 0.0696 & 2.6355 & 1.7023 \\
\hline & & $(0.000085)$ & $(0.236)$ & $(0.1139)$ & $(0.00017)$ & $(0.4909)$ & $(0.1092)$ \\
\hline
\end{tabular}


Table 6. ACL and CP of $95 \%$ CIs for $(\beta, \lambda, \mu)=(0.06,2,2)$.

\begin{tabular}{|c|c|c|c|c|c|c|c|}
\hline \multicolumn{8}{|c|}{ at constant $\alpha=1$} \\
\hline \multirow[b]{2}{*}{$\left(n, m_{1}, m_{2}\right)$} & \multirow[b]{2}{*}{ CS } & \multicolumn{2}{|c|}{ MLE } & \multirow[b]{2}{*}{$\mu$} & \multicolumn{3}{|c|}{ MCMC } \\
\hline & & $\beta$ & $\lambda$ & & $\beta$ & $\lambda$ & $\mu$ \\
\hline \multirow[t]{6}{*}{$(20,10,20)$} & I & 0.056 & 3.0358 & 4.4518 & 0.1274 & 6.0503 & 3.0825 \\
\hline & & $(0.99)$ & $(0.99)$ & $(0.99)$ & (0.99) & (0.99) & (0.99) \\
\hline & II & 0.0702 & 4.1129 & 4.1329 & 0.1779 & 5.1185 & 2.958 \\
\hline & & $(0.99)$ & $(0.99)$ & (0.99) & $(0.97)$ & $(0.98)$ & $(0.99)$ \\
\hline & III & 0.1403 & 3.9614 & 4.168 & 0.7396 & 5.9014 & 2.7929 \\
\hline & & $(0.99)$ & $(0.99)$ & $(0.99)$ & $(0.87)$ & $(0.92)$ & $(0.99)$ \\
\hline \multirow[t]{6}{*}{$(20,15,30)$} & I & 0.0549 & 3.3785 & 3.7315 & 0.1089 & 4.8809 & 2.2876 \\
\hline & & $(0.97)$ & $(0.99)$ & (0.99) & $(0.96)$ & $(0.99)$ & $(0.98)$ \\
\hline & II & 0.0579 & 3.1825 & 3.4239 & 0.1203 & 4.7176 & 2.2906 \\
\hline & & $(0.98)$ & $(0.99)$ & $(0.99)$ & $(0.97)$ & $(0.97)$ & $(0.99)$ \\
\hline & III & 0.0804 & 3.5097 & 3.8747 & 0.1964 & 5.3013 & 3.3675 \\
\hline & & $(0.99)$ & $(0.99)$ & $(0.98)$ & $(0.96)$ & $(0.99)$ & $(0.98)$ \\
\hline \multirow[t]{6}{*}{$(40,20,40)$} & I & 0.0579 & 4.2019 & 3.0721 & 0.190 & 4.2443 & 1.9591 \\
\hline & & $(0.98)$ & $(0.99)$ & $(0.98)$ & (0.99) & $(0.99)$ & $(0.99)$ \\
\hline & II & 0.0469 & 2.8267 & 2.6715 & 0.0699 & 3.2596 & 1.907 \\
\hline & & $(0.97)$ & $(0.98)$ & $(0.99)$ & $(0.98)$ & $(0.96)$ & $(0.99)$ \\
\hline & III & 0.1017 & 3.0864 & 2.9879 & 0.4108 & 4.3802 & 4.1805 \\
\hline & & $(0.99)$ & $(0.98)$ & $(0.98)$ & $(0.93)$ & $(0.97)$ & $(0.99)$ \\
\hline \multirow[t]{6}{*}{$(40,30,60)$} & I & 0.0353 & 2.1364 & 2.3602 & 0.0516 & 3.1893 & 1.5741 \\
\hline & & $(0.95)$ & $(0.99)$ & $(0.95)$ & $(0.98)$ & $(0.99)$ & $(0.98)$ \\
\hline & II & 0.0382 & 2.324 & 2.3374 & 0.0534 & 3.0202 & 1.5772 \\
\hline & & $(0.98)$ & $(0.99)$ & (0.99) & $(0.96)$ & $(0.97)$ & $(0.99)$ \\
\hline & III & 0.0525 & 2.3581 & 2.423 & 0.1017 & 3.6309 & 1.5861 \\
\hline & & $(0.99)$ & $(0.98)$ & $(0.98)$ & $(0.99)$ & $(0.97)$ & $(0.99)$ \\
\hline \multirow[t]{6}{*}{$(60,30,60)$} & I & 0.0384 & 2.4577 & 2.3284 & 0.0521 & 3.1558 & 1.5741 \\
\hline & & $(0.99)$ & $(0.99)$ & $(0.99)$ & $(0.98)$ & $(0.99)$ & $(0.99)$ \\
\hline & II & 0.0426 & 2.3196 & 2.7185 & 0.051 & 2.5739 & 1.5242 \\
\hline & & $(0.97)$ & $(0.99)$ & $(0.97)$ & (0.99) & $(0.98)$ & $(0.99)$ \\
\hline & III & 0.0788 & 2.4596 & 2.4006 & 0.2046 & 3.4947 & 1.5811 \\
\hline & & (0.99) & $(0.99)$ & $(0.98)$ & $(0.97)$ & $(0.96)$ & $(0.99)$ \\
\hline \multirow[t]{6}{*}{$(60,50,90)$} & I & 0.0301 & 1.8135 & 1.972 & 0.0386 & 2.4831 & 1.1937 \\
\hline & & $(0.95)$ & $(0.99)$ & $(0.93)$ & $(0.95)$ & $(0.99)$ & $(0.97)$ \\
\hline & II & 0.0279 & 1.5825 & 1.8247 & 0.0387 & 2.3351 & 1.2341 \\
\hline & & $(0.99)$ & $(0.99)$ & $(0.96)$ & (0.99) & (0.999) & $(0.99)$ \\
\hline & III & 0.0328 & 1.589 & 1.7172 & 0.0553 & 2.7334 & 1.2375 \\
\hline & & $(0.98)$ & $(0.96)$ & $(0.95)$ & $(0.96)$ & $(0.98)$ & $(0.96)$ \\
\hline
\end{tabular}


Table 7. AVG and MSE of ML and Bayes estimates for $(\alpha, \lambda, \mu)=(10,2,2)$.

\begin{tabular}{|c|c|c|c|c|c|c|c|}
\hline \multicolumn{8}{|c|}{ at $\operatorname{constant} \beta=1$} \\
\hline \multirow[b]{2}{*}{$\left(n, m_{1}, m_{2}\right)$} & \multirow[b]{2}{*}{ CS } & \multicolumn{2}{|r|}{ MLE } & \multirow[b]{2}{*}{$\mu$} & \multicolumn{3}{|c|}{ MCMC } \\
\hline & & $\alpha$ & $\lambda$ & & $\alpha$ & $\lambda$ & $\mu$ \\
\hline \multirow[t]{6}{*}{$(20,10,20)$} & I & 10.7008 & 2.501 & 1.8418 & 11.0046 & 2.5956 & 2.0397 \\
\hline & & (1.2898) & $(0.3345)$ & $0.0978)$ & (1.8442) & $(0.4541)$ & $(0.1124)$ \\
\hline & II & 10.376 & 2.6447 & 1.6149 & 10.4635 & 2.7214 & 1.7827 \\
\hline & & $(0.7166)$ & $(0.6086)$ & $(0.2041)$ & $(0.844)$ & $(0.7559)$ & $(0.1231)$ \\
\hline & III & 10.6219 & 3.0596 & 1.5332 & 10.8375 & 3.4989 & 1.715 \\
\hline & & $(0.9651)$ & $(1.4262)$ & $(0.3006)$ & $(1.7516)$ & $(3.2126)$ & $(0.2077)$ \\
\hline \multirow[t]{6}{*}{$(20,15,30)$} & I & 10.3427 & 2.2998 & 1.8241 & 10.5203 & 2.3462 & 1.9452 \\
\hline & & $(0.794)$ & (0.1719) & $(0.0891)$ & (1.0157) & $(0.2208)$ & $(0.0723)$ \\
\hline & II & 10.3454 & 2.4789 & 1.7113 & 10.4634 & 2.5291 & 1.8254 \\
\hline & & $(0.7494)$ & $(0.341)$ & $(0.1481)$ & $(0.900)$ & $(0.424)$ & (0.1079) \\
\hline & III & 10.3828 & 2.6167 & 1.657 & 10.5635 & 2.745 & 1.7776 \\
\hline & & $(0.7039)$ & $(0.5117)$ & $(0.1732)$ & (1.0712) & $(0.7482)$ & $(0.1213)$ \\
\hline \multirow[t]{6}{*}{$(40,20,40)$} & I & 10.3307 & 2.3218 & 1.8209 & 10.4596 & 2.3476 & 1.9183 \\
\hline & & (0.6479) & $(0.1802)$ & $(0.0822)$ & $(0.7872)$ & $(0.2073)$ & $(0.0674)$ \\
\hline & II & 10.387 & 2.5788 & 1.6489 & 10.4492 & 2.6152 & 1.7334 \\
\hline & & $(0.6155)$ & $(0.4786)$ & $(0.1862)$ & $(0.6802)$ & $(0.5303)$ & $(0.1460)$ \\
\hline & III & 10.4848 & 2.8496 & 1.5301 & 10.6181 & 3.033 & 1.6122 \\
\hline & & (0.9172) & $(0.9353)$ & $(0.2759)$ & (1.192) & (1.3709) & $(0.2161)$ \\
\hline \multirow[t]{6}{*}{$(40,30,60)$} & I & 10.2877 & 2.2525 & 1.8005 & 10.3642 & 2.2696 & 1.8594 \\
\hline & & $(0.6171)$ & $(0.1206)$ & $(0.0765)$ & (0.7097) & (0.1349) & $(0.0621)$ \\
\hline & II & 10.4576 & 2.445 & 1.7121 & 10.5141 & 2.4645 & 1.771 \\
\hline & & $(0.8037)$ & $(0.2925)$ & $(0.1167)$ & $(0.8842)$ & $(0.3259)$ & $(0.0906)$ \\
\hline & III & 10.3383 & 2.3375 & 1.7607 & 10.436 & 2.3857 & 1.8082 \\
\hline & & $(0.5735)$ & $(0.1707)$ & $(0.0881)$ & $(0.6961)$ & $(0.223)$ & $(0.0706)$ \\
\hline \multirow[t]{6}{*}{$(60,30,60)$} & I & 10.1902 & 2.2849 & 1.8004 & 10.2894 & 2.3057 & 1.8637 \\
\hline & & $(0.5777)$ & $(0.1403)$ & $(0.0811)$ & $(0.6584)$ & (0.1588) & $(0.0662)$ \\
\hline & II & 10.2647 & 2.5227 & 1.6399 & 10.3048 & 2.5475 & 1.6921 \\
\hline & & $(0.4723)$ & $(0.3675)$ & $(0.1619)$ & (0.4974) & $(0.405)$ & $(0.1292)$ \\
\hline & III & 10.2817 & 2.3968 & 1.746 & 10.3032 & 2.4313 & 1.8091 \\
\hline & & $(0.6363)$ & $(0.2158)$ & $(0.1016)$ & (0.6973) & $(0.2794)$ & $(0.0766)$ \\
\hline \multirow[t]{6}{*}{$(60,50,90)$} & I & 10.1694 & 2024 & 1.8155 & 10.2176 & 2.2495 & 1.853 \\
\hline & & $(0.5023)$ & $(0.0986)$ & $(0.0697)$ & $(0.5666)$ & $(0.1082)$ & $(0.0609)$ \\
\hline & II & 10.1431 & 2.2866 & 1.7472 & 10.1724 & 2.2966 & 1.7797 \\
\hline & & $(0.4156)$ & $(0.1393)$ & $(0.0873)$ & $(0.4323)$ & $(0.1486)$ & $(0.0734)$ \\
\hline & III & 10.4057 & 2.3178 & 1.7909 & 10.4669 & 2.3402 & 1.8256 \\
\hline & & (0.7198) & $(0.152)$ & $(0.0785)$ & (0.7897) & $(0.1711)$ & $(0.0694)$ \\
\hline
\end{tabular}


Table 8. ACL and CP of $95 \%$ CIs for the parameters $(\alpha, \lambda, \mu)=(10,2,2)$

\begin{tabular}{|c|c|c|c|c|c|c|c|}
\hline \multicolumn{8}{|c|}{ at constant $\beta=1$} \\
\hline \multirow[b]{2}{*}{$\left(n, m_{1}, m_{2}\right)$} & \multirow[b]{2}{*}{ CS } & \multicolumn{2}{|c|}{ MLE } & \multirow[b]{2}{*}{$\mu$} & \multicolumn{3}{|c|}{ MCMC } \\
\hline & & $\alpha$ & $\lambda$ & & $\alpha$ & $\lambda$ & $\mu$ \\
\hline \multirow[t]{6}{*}{$(20,10,20)$} & I & 11.1008 & 5.3223 & 3.9355 & 9.0397 & 3.7072 & 3.2527 \\
\hline & & $(0.99)$ & $(0.99)$ & $(0.99)$ & (0.99) & $(0.99)$ & $(0.99)$ \\
\hline & II & 6.7916 & 4.2454 & 3.6891 & 7.6956 & 4.183 & 2.8243 \\
\hline & & $(0.99)$ & $(0.99)$ & $(0.98)$ & $(0.97)$ & $(0.99)$ & $(0.99)$ \\
\hline & III & 6.0575 & 5.1354 & 3.7134 & 9.7149 & 7.8724 & 2.7028 \\
\hline & & (0.99) & $(0.98)$ & $(0.98)$ & $(0.99)$ & (0.995) & (0.993) \\
\hline \multirow[t]{6}{*}{$(20,15,30)$} & I & 9.9457 & 3.3441 & 3.6462 & 7.1839 & 2.555 & 2.4516 \\
\hline & & (0.992) & (0.994) & (0.995) & (0.991) & (0.998) & (0.996) \\
\hline & II & 7.5563 & 3.6064 & 3.1659 & 6.9438 & 2.9543 & 2.2971 \\
\hline & & (0.998) & $(0.99)$ & (0.997) & (0.999) & $(0.995)$ & (0.991) \\
\hline & III & 6.9475 & 3.5343 & 3.1699 & 7.9778 & 3.774 & 2.2567 \\
\hline & & (0.999) & (0.996) & (0.993) & $(0.995)$ & (0.994) & (0.999) \\
\hline \multirow[t]{6}{*}{$(40,20,40)$} & I & 6.8394 & 2.559 & 2.7329 & 5.7899 & 2.2057 & 2.0694 \\
\hline & & $(0.99)$ & $(0.99)$ & $(0.99)$ & $(0.99)$ & $(0.99)$ & $(0.99)$ \\
\hline & II & 4.9991 & 2.9631 & 2.9531 & 5.4599 & 2.7755 & 1.8767 \\
\hline & & $(0.98)$ & $(0.99)$ & $(0.96)$ & $(0.98)$ & $(0.99)$ & $(0.98)$ \\
\hline & III & 4.922 & 3.436 & 3.0587 & 6.8644 & 4.4326 & 1.7545 \\
\hline & & $(0.92)$ & $(0.99)$ & $(0.89)$ & $(0.99)$ & $(0.98)$ & $(0.94)$ \\
\hline \multirow[t]{6}{*}{$(40,30,60)$} & I & 6.8264 & 2.2883 & 2.4979 & 4.989 & 1.7294 & 1.6202 \\
\hline & & $(0.99)$ & $(0.98)$ & $(0.97)$ & $(0.99)$ & $(0.99)$ & $(0.99)$ \\
\hline & II & 5.1768 & 2.345 & 2.2533 & 4.8322 & 2.0064 & 1.5289 \\
\hline & & $(0.98)$ & $(0.99)$ & $(0.99)$ & $(0.98)$ & $(0.99)$ & $(0.99)$ \\
\hline & III & 7.6706 & 2.6993 & 2.4883 & 5.3526 & 2.1018 & 1.5218 \\
\hline & & $(0.99)$ & $(0.99)$ & $(0.99)$ & $(0.98)$ & $(0.99)$ & $(0.98)$ \\
\hline \multirow[t]{6}{*}{$(60,30,60)$} & I & 6.5778 & 2.4444 & 2.5063 & 4.7873 & 1.7859 & 1.6208 \\
\hline & & $(0.99)$ & $(0.99)$ & $(0.98)$ & $(0.97)$ & $(0.99)$ & $(0.99)$ \\
\hline & II & 3.8321 & 2.2191 & 2.2521 & 4.3144 & 2.1903 & 1.4579 \\
\hline & & $(0.98)$ & $(0.99)$ & $(0.98)$ & (0.99) & $(0.97)$ & $(0.98)$ \\
\hline & III & 6.472 & 2.4208 & 2.8273 & 5.0385 & 2.5281 & 1.5586 \\
\hline & & $(0.99)$ & $(0.99)$ & $(0.99)$ & $(0.98)$ & $(0.97)$ & $(0.99)$ \\
\hline \multirow[t]{6}{*}{$(60,50,90)$} & I & 5.7573 & 1.6062 & 2.3605 & 3.9548 & 1.3452 & 1.2847 \\
\hline & & $(0.99)$ & $(0.99)$ & $(0.98)$ & $(0.99)$ & $(0.98)$ & $(0.99)$ \\
\hline & II & 4.6031 & 1.5695 & 2.2536 & 3.7996 & 1.4078 & 1.228 \\
\hline & & $(0.99)$ & $(0.97)$ & $(0.98)$ & $(0.99)$ & $(0.95)$ & $(0.98)$ \\
\hline & III & 5.6451 & 1.644 & 2.0888 & 4.2791 & 1.550 & 1.2402 \\
\hline & & $(0.99)$ & $(0.99)$ & $(0.98)$ & $(0.99)$ & $(0.98)$ & $(0.97)$ \\
\hline
\end{tabular}




\section{Concluding remarks}

In this paper,we considered the maximun likelihood, bootstrap and Bayes estimats for the parameters of the MWD. This paper also studied the construction of confidence intervals for the parameters. We use MCMC method because we donot get explicit form for the Bayes estimate. A numerical example using simulated data is presented to show how the MCMC and parametric bootstrap methods work based on PRO-II-C. Finally, a simulation study was performed to compare the performance of MLE and Bayes estimates for different sample sizes. The following conclusions can be drawn from Tables 4, 5, 6, 7 and 8:

(i) From Table 4, we notice that Scheme I is the best in the sense of having smaller MSEs for fixed values of $n, m_{1}, m_{2}$.

(ii) From Table 5, we observe that at constant $\alpha$, MLEs have smaller MSE than Bayes estimates.

(iii) It is observed from Table 7 that when the sample size increases, the MSEs decreases.

(iv) From Table 7, it can be seen that for parameters $\alpha$ and $\lambda$, MLEs gives more accurate results then Bayes estimates in the sense of having smaller MSEs, but for the parameter $\mu$, Bayes estimates are better in MSEs than MLEs.

(v) From Table 8, it can be seen that CRIs show more accurate results than the ACIs because the lengths of CRIs are less than the lengths of ACIs.

\section{Acknowledgement}

The authors are grateful to the anonymous referee for a careful checking of the details and for helpful comments that improved this paper.

\section{References}

[1] M. V. Aarset, How to identify a bathtub hazard rate. IEEE Transactions on Reliability 36 (1987) 106108.

[2] A. H. Abdel-Hamid, and E. K. Al-Hussaini, Progressive stress accelerated life tests under finite mixture models. Metrika. 66 (2007) 213-231.

[3] E. K. AL-Hussaini and A. H. Abdel-Hamid, Accelerated life tests under finite mixture models. Statistical Computation and Simulation 76(8) (2006) 673-690.

[4] E. K. AL-Hussaini and A. H. Abdel-Hamid, Bayesian estimation of the parameters, Reliability and Hazard rate functions of mixtures under accelerated life tests. Communications in Statistics - Simulation and Computation 33(4) (2004) 963-982.

[5] V. Bagdonavicius and M. Nikulin, Accelerated Life Models, Modeling and Statistical Analysis. Boca Raton, FL, Chapman \& Hall/CRC Press, (2002).

[6] D. S. Bai and M. S. Kim and S. H. Lee, Optimum simple step-stress accelerated life tests with censoring. IEEE Transactions on Reliability 38(5) (1989) 528-532.

[7] N. Balakrishnan and R. Aggarwala, Progressive Censoring: Theory, Methods and Applications. Birkhauser Boston, (2000).

[8] N. Balakrishnan, and R. A. Sandhu, A simple simulation algorithm for generating progressively type-II censored samples. American Statistical Association 49 (1995) 229-230.

[9] Z. Chen, A new two-parameter lifetime distribution with bathtub shape or increasing failure rate function. Statistics and Probability Letters 49 (2000) 155-161.

[10] A. C. Davison and D. V. Hinkley, Bootstrap Methods and Their Applications. 2nd ed. Cambridge: Cambridge University Press, (1997).

[11] B. Efron and The jackknife, The Bootstrap and Other Resampling Plans. Philadelphia, SIAM, (1982).

[12] B. Efron and R. J. Tibshirani, An Introduction To the Bootstrap. NewYork, Chapman and Hall (1993). 
[13] R. M. EL-Sagheer and M. Ahsanullah, Statistical inference for a step-stress partially accelerated life test model based on progressively type-II censored data from lomax distribution. Applied Statistical Science 21(4) (2015) 307-323.

[14] T. H. Fan, W. L. Wang and N. Balakrishnan, Exponential progressive step-stress life-testing with link function based on Box Cox transformation. Statistical Planning and Inference 138 (2008) 2340-2354.

[15] A. Gupta, B. Mukherjee and S. K. Upadhyay, Weibull extension: A Bayes study using Markov chain Monte Carlo simulation. Reliability Engineering and System Safety 93 (2008) 1434-1443.

[16] P. Hall, Theoretical comparison of bootstrap confidence intervals. Annals of Statistics 16 (1988) 927953.

[17] A. A. Ismail, A. A. Abdel-Ghalyb and E. H. El-Khodary, Optimum constant-stress life test plans for Pareto distribution under type-I censoring. Statistical Computation and Simulation 81(12) (2011) 18351845.

[18] C. M. Kim and D. S. Bai, Analysis of accelerated life test data under two failure modes. International Journal of Reliability, Quality and Safety Engineering 9 (2002) 111-125.

[19] C. D. Lai, Generalized Weibull Distribution. Springer, (2014).

[20] H. M. Ma and W. Q. Meeker, Optimum step-stress accelerated life test plans for log-location-scale distributions.Naval Research Logistics 55 (2008) 551-562.

[21] W. Q. Meeker and L. A. Escobar, Statistical Methods for Reliability Data. New York, Wiley \& Sons, (1998).

[22] N. Metropolis, A. W. Rosenbluth, M. N. Rosenbluth, A. H. Teller and E. Teller, Equations of state calculations by fast computing machines. Journal of Chemical Physics 21 (1953) 1087-1091.

[23] R. Miller and W. B. Nelson, Optimum simple step-stress plans for accelerated life testing. IEEE Transactions on Reliability 32 (1983) 59-65.

[24] W. Nelson, Accelerated Testing, Statistical Models, Test Plans and Data Analysis. NewYork, Wiley, (1990).

[25] W. Nelson, Residuals and their analysis for accelerated life tests with step and varying stress. IEEE Transactions on Reliability 57 (2008) 360-368.

[26] C. P. Robert and G. Casella, Monte Carlo Statistical Methods. NewYork, Springer,(2004).

[27] R. M. Smith and L. J. Bain, An exponential power life-testing distribution. Communications in Statistics-Theory and Methods 4 (1975) 469-481.

[28] Y. Tangi, Q. Guani, P. Xu and H. Xu, Optimum design for type-I step-stress accelerated life tests of two-parameterWeibull distributions. Communications in Statistics - Theory and Methods 41 (2012) 3863-3877.

[29] Y. Tang, M. Xie and T. N. Goh, Statistical analysis of a Weibull extension model. Communications in Statistics-Theory and Methods 32(5) (2003) 913-928.

[30] S. A. VanderWiel and W. Q. Meeker, Accuracy of approx confidence bounds using censored Weibull regression data from accelerated life tests. IEEE Transactions on Reliability, 39(3) (1990) 346-351.

[31] R. Wang and H. Fei, Statistical inference of Weibull distribution for tampered failure rate model in progressive stress accelerated life testing. Journal of Systems Science and Complexity 17 (2004) 237243.

[32] S. J. Wu, Y. P. Lin and S. T. Chen, Optimal step-stress test under type I progressive group-censoring with random removals. Statistical Planning and Inference 138 (2008) 817-826.

[33] M. Xie, Y. Tang and T. N. Goh, A modified Weibull extension with bathtub-shaped failure rate function. Reliability Engineering and System Safety 76 (2002) 279-285. 